\title{
Histoire d'une amitié : correspondance intime entre Chapleau et DeCelles (1876-1898) (suite et fin)
}

\section{Jacques Gouin}

Volume 18, numéro 4, mars 1965

URI : https://id.erudit.org/iderudit/302416ar

DOI : https://doi.org/10.7202/302416ar

Aller au sommaire du numéro

Éditeur(s)

Institut d'histoire de l'Amérique française

ISSN

0035-2357 (imprimé)

1492-1383 (numérique)

Découvrir la revue

Citer cet article

Gouin, J. (1965). Histoire d'une amitié : correspondance intime entre Chapleau et DeCelles (1876-1898) (suite et fin). Revue d'histoire de l'Amérique française, 18(4), 541-565. https://doi.org/10.7202/302416ar d'utilisation que vous pouvez consulter en ligne. 


\title{
HISTOIRE D'UNE AMITIÉ :
}

\section{Correspondance intime entre Chapleau et DeCelles}

\author{
(1876-1898) \\ (suite et fin)*
}

Le 26 mai 1893, DeCelles faisait part encore une fois à son ami des attaques malveillantes dont celui-ci était l'objet dans les journaux, ainsi que des nouvelles d'Ottawa:

C'est étonnant comme on continue à s'occuper de toi au pays. MacDonnell et Tarte lancent des cancans et les autres journaux les gobent. Je n'ai rien écrit sur les affaires du Manitoba. Je ne suis pas assez au courant de ce qui s'est fait. Inutile de compromettre la situation, rien ne pressant. J'écrirai un article sur toi à un autre point de vue.

Les journaux libéraux livrent un assaut terrible au gouvernement que personne ne défend, pas même la Minerve, pas même le Courrier du Canada. Ils ont cependant la partie belle. Les libéraux font tant de bêtises. Leur sympathie pour la Canada-Revue, leur aliène le bon-vouloir du clergé, qui serait heureux de ne pas croire après cela, à leur zèle pour les catholiques du Manitoba, si on le lui faisait voir, mais voilà le diable, on ne dit rien. Il est temps que sir John Thompson revienne. Peut-être fera-t-il sortir la presse de sa torpeur. Pour cela, il faut de l'argent et il n'y a pas ce fonds de reptiles. Les mœurs politiques changent. La pauvreté fait peur à tout le monde. On ne sait plus être pauvre et honorable comme les anciens.

Eusèbe Sénécal voudrait avoir le contrôle absolu de la Minerve, pour en faire un journal comme le Monde sans doute, à vendre six fois la semaine. C'est sujet.

* Voir notre Revue, XVIII: 363-386, pour le premier article sur ce 
pourtant un des moins ardents. Ils en verront bien un autre le jour où paraîtra le Soleil de Louison. Le voilà un astre qui ne donnerait pas ses rayons pour rien .... 1 .

Chapleau se rétablissait alors d'une maladie, ce qui explique sans doute son silence à cette époque:

Tu ne nous accables pas de lettres. Tout le monde demande de tes nouvelles et personne n'en a à donner. On pourrait conclure que tu n'es pas mieux, mais les journaux disent que tu as dîné chez lord Dufferin, donc tu es mieux. Si tu peux tenir une fourchette, il doit t'être facile de lever une plume.

Ici, on se prépare à la lutte. Les libéraux vont venir à Ottawa la semaine prochaine. Très crâne leur idée de tenir leur conférence à la barbe des ministres, à deux pas de ces bureaux qu'ils convoitent tant. S'ils allaient manquer leur affaire par exemple, ils auront joliment prêté le flanc au ridicule. D'après ce que je puis voir, il n'y aura qu'une planche dans leur plate-forme [a plank in their platform, américanisme qui a eu la vie dure]. Réforme du tarif sur les bases de la réciprocité commerciale. C'est un grand embarras qu'ils se mettent sur les bras pour le jour de leur triomphe. C'est très mal de promettre une réforme dont la réalisation dépend d'un pays étranger. Il ne sera pas question à la convention ni des écoles du Manitoba ni d'autres questions qui pourraient diviser les libéraux, aussi susceptibles d'éparpillement que les conservateurs.

Ceux-ci ont eu une petite réunion dont le résultat paraît être d'avoir fait éclater au grand jour des divisions latentes. Ouimet et ses amis veulent mettre la main sur la Minerve, que sir A.P.C. [Caron] ne veut pas lâcher, dit-on.

Ouimet et ses amis attendent le retour de sir John Thompson pour qu'il force la main à sir Adolphe. Celui-ci a dû te dire qu'il trouve que Ouimet conduit la barque bien mal, à Montréal. Ce sera une raison pour l'empêcher de lâcher la Minerve.

1 DeCelles à Chapleau, 26 mai 1893. 
Celle-ci devient de plus en plus l'organe de sa direction [souligné par DeCelles], comme disait l'Opinion publique l'autre jour. C'est assez cela, n'est-ce pas ? Tassé a l'air de croire qu'il n'y a plus que lui qui vaille la peine d'être défendu. Il part prochainement pour aller remplir Chicago de son importance.

Je partirai fini juin pour l'humble Malbaie où j'occuperai un modeste cottage à l'ombre de la Villa Taché qui est superbe, me dit-on. J'ai hâte de travailler au frais. Il fait rudement chaud à Ottawa.

J'ai vu Mad. Pope qui m'a donné de vos nouvelles. Ce qu'elle a dû scandaliser les ladies Thompson, Caron \& Tupper avec ses allures si peu officielles, si la moitié de ce qu'elle me dit avoir fait, est vrai ${ }^{2}$ !

Le 25 septembre 1893, à Chapleau, qui lui avait adressé son profil coulé en bronze, DeCelles écrit ce qui suit:

Le médaillon et la médaille sont arrivés samedi. Merci pour la bibliothèque et pour moi. Ton profil de bronze est accroché à la place d'honneur dans mon foyer.

Si jamais je suis coulé en métal, à titre de gouverneur d'une île encore inconnue, je te ferai l'hommage de ma tête. Comme cet acte de réciprocité pourrait se faire attendre, je t'offre mes remerciements pour le moment.

Je t'envoie un discours de lord Salisbury. Je n'en ai jamais lu de plus propre à donner une idée de sa manière, de son ironie brutale, de sa méchanceté de singe ${ }^{3}$.

Le 25 novembre 1894, se rappelant que vingt ans exactement auparavant DeCelles avait servi de maître des cérémonies au mariage de son ami, il lui adresse la lettre spirituelle et fine que voici :

Ton discours du trône et tout le bruit qui s'est fait à Québec à l'ouverture du Parlement ne sauraient me faire oublier qu'il y a vingt ans, - le 25 nov., - j'assistais en qualité de maître des céré-

2 Ibid., 9 juin 1893.

3 Ibid., 25 sept. 1893. 
monies (peu stylé) à un événement capital de ta vie. C'est là un anniversaire que je ne puis laisser passer inaperçu car il me rappelle des souvenirs agréables et aussi le rôle important que j'ai joué, t'ayant conduit deux fois à l'autel, afin d'être bien certain que tu étais solidement marié. Rien d'étonnant, - à part d'autres raisons, - que le nœud ait bien tenu et si longtemps. Mon succès, - Madame Chapleau et toi y aidant beaucoup, - aurait dû m'engager dans la match-making. Dans tous les cas, les souhaits que je faisais le 25 nov. 1874, se sont plus que réalisés. Tu as gravi tous les échelons des honneurs auxquels un citoyen de notre province peut aspirer. Aujourd'hui, te voilà à la tête d'un million et demi de fidèles sujets, que tu traites très bien, s'il est vrai que tu leur prêtes de l'argent; c'est là le comble de l'amabilité pour un père de famille. Aussi tous t'adorent, à part un petit nombre guidé par une mauvaise Etoile [allusion à un journal de l'époque]. S'ils se montrent enfants mal élevés, on ne te rend pas heureusement responsable de leur mince instruction et de leur absence d'éducation.

Nous avons joliment changé toi et moi depuis l'heureuse date en question; nos cheveux sont devenus blancs... ou rares, mais consolons-nous; nous devons être vingt fois plus sages qu'au temps jadis. Seule Madame Chapleau, - qui n'avait pas de sagesse à acquérir, tenant du Ciel tous ses plus heureux dons, - est restée la même. Je la revois encore à Sherbrooke lorsque je lui fus présenté comme si c'était hier. Elle se ressemble à un degré singulier. C'est ce que nous disons souvent moi et ma femme, qui unit ses félicitations aux miennes pour vous les offrir.

Nous prions ste Catherine, patronne de bien des bonnes choses, qu'elle étire le plus possible la durée d'une des plus heureuses unions conclues sous ses auspices ${ }^{4}$.

Le ton de cette lettre me paraît extrêmement touchant, à un double point de vue: il dénote d'abord un sentiment sincère d'amitié indéfectible, et ensuite une sorte de sérénité légèrement

4 Ibid., 25 nov. 1894. 
désabusée vis-à-vis la chose politique, je devrais dire, plus précisément vis-à-vis ce vieux parti conservateur qui, après avoir présidé à la naissance de la Confédération, se trouve à la veille d'être emporté dans la tourmente des élections de 1896. D'ailleurs, voici ce qu'ajoute DeCelles quelques mois plus tard:

... Je ne crois pas aux élections et n'y ai jamais cru. J'ai été seul de mon avis pendant longtemps, mais aujourd'hui il est partagé par bien des gens. Personne ne les désire chez les ministres, sauf Wallace et Wood, qui redoutent la politique du gouvernement relativement aux écoles du Manitoba, si le jugement du C.P. [Conseil privé] donne gain de cause aux catholiques du Manitoba car le gouvernement sera bien forcé d'appliquer la loi remédiatrice, sans toucher à la législation Greenway que le jugement n'atteindra pas ... Puis, une campagne, l'hiver, c'est moins que plaisant. Tout le monde a horreur du froid, ennemi des gosiers et fabri [sic] de bronchites. $\mathrm{Au}$ reste, Bowell n'a pas encore retrouvé sa voix (ni trouvé sa voie non plus, je pense) et dans ce statu quo, rien ne peut être décidé, pas même la date de la convocation des chambres. Je regrette d'apprendre que tu es revenu de VillaMaria avec un mauvais rhume. Je suppose que le mouvement cadencé des robes frou-froutantes de ces demoiselles faisant le plongeon d'honneur aura jeté un froid dans la salle, et de là ton refroidissement et ta bronchite. Je pense bien, comme toi, que les campagnes d'hiver te sont à jamais interdites, si tu reviens dans la politique. Tu as assez d'autorité pour te passer du contact direct avec le peuple. Ton moyen d'action devra être la presse aux mille voix. Tu devras parler de haut et tu seras entendu de loin. Au reste, le husting te sera ouvert l'été s'il te reste du goût pour les pow-wows populaires, à grand orchestre. Je t'engage à lire dans la Revue des 2 Mondes les articles du duc de Broglie sur les préliminaires de la guerre de Sept ans. C'est du plus haut intérêt... ${ }^{5}$

Chapleau, comme on le sait, ne reviendra jamais plus à la politique active. Mais son ami n'en espère pas moins toujours

5 Ibid., 24 jan. 1895. 
qu'il se décidera un jour à reprendre en mains les destinées du parti conservateur qui paraît voué à un désastre imminent. On sent d'ailleurs que DeCelles ne se fait plus d'illusions, si ce n'est que sa foi en Chapleau reste inébranlable:

... Les oreilles ont dû te tinter le soir de la démonstration du Parc Sohmer. Tarte est toujours le même audacieux politicien, risquant le tout pour le tout. C'est ça qui a dû faire faire une tête à tes anciens collègues d'ici, toujours très inquiets non au sujet de ta santé, mais de ton attitude. Vingt fois, Caron m'a demandé ce que tu allais faire et autant de fois je lui ai répondu: "Il est bien à Spencer Wood; mon avis est qu'il va y rester." Il est persuadé que tu as une dent contre Ouimet et que tu n'as pas un râtelier contre lui. Ouimet a entendu bien des cancans à Montréal. Il n'y croit pas. Je lui ai dit que c'était trop bête... Il a fini par dire: "Il pourrait bien avoir des relations avec les rouges, mais c'est probablement pour les jouer, comme il a déjà fait." Je ne le vois que très peu. Il passe une fois par mois à mon bureau ...

La semaine dernière, Bowell paraissait décidé à avoir une session. Aujourd'hui, le vent est aux élections. Rien ne sera décidé, je crois, ce mois-ci. D'après ce que j'entends dire, le gouvernement est disposé à rendre justice aux catholiques. Tupper n'a pas caché sa pensée sur ce point. Il a dit à un reporter... "that he was prepared to make a report on the lines of the P.C.'s decision"... Cette décision serait un grand pas. Elle n'aurait qu'un défaut: celui de venir trop tard, une fois le courant d'opinion formé contre le gouvernement. Je n'ai jamais vu inertie pareille à celle de nos ministres français [sic]. Angers ne paraît s'occuper que d'obtenir une rétractation de l'Electeur; Ouimet planait à N.Y. pendant qu'on le démolissait à Montréal; quant à Caron, c'est l'homme le plus occupé du monde à ne rien faire.

Tout le monde dit: "Ah ! si Chapleau était ici, tout irait autrement." You are in great demand, comme disent les Anglais. Ce qui peut consoler les ministres, c'est le vague de la politique de leurs 
adversaires qui dévoile les dessous de leur administration interne. Il y a de la tire: on sent les dissentiments qui éclateront après l'arrivée de Laurier au pouvoir. Il n'y a pas que le parti conservateur qui soit pauvre en hommes. Mais la grande force des libéraux en ce moment, c'est leur foi en leur triomphe futur. Je ne leur ai jamais vu tant d'enthousiasme: jamais non plus leurs espérances n'ont été plus fondées ${ }^{6}$.

DeCelles se montrait là très perspicace, puisque, en effet, les jours du parti conservateur étaient comptés. Ce qui nous frappe ici, dans toute cette correspondance, c'est l'aveuglement tenace de l'esprit de parti. Aveuglement, pourrait-on dire, qui a toujours été l'obstacle majeur à une solidarité véritable et profonde chez les Canadiens français. Mais, c'est là le sort, le drame, - de toute minorité ethnique au sein d'une fédération. On a toujours l'impression que les hommes politiques, dans de telles conditions, si bien intentionnés qu'ils soient, ne font que s'agiter comme des marionnettes dont les fils sont tirés par ceux qui représentent la majorité. Si c'est vrai encore aujourd'hui, c'est dire à quel point ce l'était à l'époque de Chapleau et de DeCelles. Voyons encore ce jeu de "marionnettes" dans les lignes que voici:

Rien n'est encore décidé au sujet des élections, me dit Angers. C'en a bien l'air cependant. Les ministres canadiens [-français] n'en veulent pas, leurs amis non plus, et une foule de députés d'Ontario sont de leur avis. L'indemnité leur irait bien avant la lutte. Ce sont les ministres d'Ontario qui sont les plus ardents à la bataille. Ils voudraient revenir de la lutte avant l'exécution du jugement [sur la question des écoles du Manitoba]. Ils ont tellement dit à leurs amis que le Gouvernement n'interviendrait pas à [sic] Manitoba qu'ils ne savent plus comment s'y prendre pour leur administrer la pilule ?.

Quelques semaines plus tard, le jeu se poursuivait toujours:

6 Ibid., 20 fév. 1895.

7 Ibid., 2 mars 1895. 
Je viens de voir Girouard, qui, tout en fumant un cigare à mon bureau, m'a mis au courant de ce qui s'est passé au conseil depuis quelques jours. La lutte a été vive, très vive; hier, à 3 heures, tout paraissait perdu. Les ministres canadiens [-français] étaient prêts à résigner [démissionner] tous trois, - même Caron, ce qui va te surprendre. Girouard prétend qu'il était le plus déterminé à tout casser. Il était à cheval, quoi ! Ils sont sortis du conseil, se tenant par le bras... Bowell voulait d'un ordre en conseil [décret], d'un ton conciliant, mais nos gens ont insisté pour avoir un ordre au Parlement du Manitoba, - non pas au gouvernement, - d'avoir à modifier sa législation dans le sens du jugement du conseil privé.

Je ne crois pas que depuis la Confédération, des ministres d'ici se soient trouvés à pareille fête. Ce qui a donné du nerf à Caron, c'est que Bowell l'a très irrité, en allant deux fois à Québec sonder l'opinion sans le consulter. Ce manque d'égards l'a complètement exaspéré.

L'affaire est loin d'être réglée; ça commence seulement. Je suis persuadé que Manitoba ne fera rien, ce qui paraît être une vérité de La Palisse. Il me semble que nos gens de là-bas n'obtiendront justice que lorsqu'ils seront assez nombreux, assez unis pour faire sentir leur influence là-bas dans les élections locales, à moins que Laurier arrivant au pouvoir n'obtienne des concessions de Greenway, à titre gracieux. Je ne crois [pas] qu'un parlement conduit soit par Laurier soit par Bowell consente à l'intervention dans les affaires provinciales, même avec l'appui du jugement d'outre-mer... Il y a dans tout ce que je viens de te dire, bien des choses qui sont de nature confidentielle. Tu sauras les garder comme je te les donne pour ton édification ${ }^{8}$.

Et pour la nôtre, pourrait-on ajouter, car, au fond, la situation a-t-elle tellement changé depuis 1895? Par voie de compensation, toutefois, on cherchait, comme aujourd'hui encore, à "caser" certains Canadiens français éminents dans le monde des lettres ou de l'érudition, comme en fait foi cette lettre.

8 Ibid., 20 mars 1895. 
... Angers m'a demandé d'offrir à J.-E. Roy la place de Marmette, aux archives. Roy a refusé. J'ai envie de lui suggérer Myrand, de Québec, placé à la Cour, si tu crois que Taillon pourrait placer Delpit [secrétaire particulier de Chapleau] à ce dernier endroit. Il serait plus simple, me diras-tu, d'envoyer Delpit à Ottawa, mais Angers voudrait-il nommer un Français, et un homme nullement au fait de l'histoire du Canada? C'est ce dont je doute; si tu vois une autre combinaison, dis-le moi au plus tôt.

Nous avons beaucoup regretté que Faucher ne soit pas venu à la société royale. Prévost, qui l'avait remis sur pied, il y a trois ans, se proposait de le retaper à neuf encore une fois. Faucher m'écrit qu'il est trop pauvre pour venir ici. Chose singulière, lorsqu'il n'émergeait [n'émargeait ?] pas et qu'il était par conséquent sans le sou, ou censé l'être, il ne manquait pas une séance de notre affaire, et aujourd'hui qu'il a un assez joli salaire (pour un garçon) nous ne le voyons plus. Je commence à croire que c'est ton absence d'Ottawa qui l'empêche de venir.

Je me suis amusé ce matin, à voir dans le Courrier, le plan d'un discours qu'il te suggère pour souhaiter la bienvenue au marquis de Lévis. Ces messieurs du Courrier feraient mieux de s'occuper de la besogne de leur journal, peu fait, que de s'inquiéter de celle des autres qui ont l'habitude de pas mal s'en tirer.

Ton voisin Lemoine [sir James McPherson] te donnera sans doute des nouvelles d'Ottawa ${ }^{9}$.

Une semaine plus tard, Chapleau répondait à cette lettre pour proposer cette autre "combinaison":

J'ai parlé de cette affaire de Myrand et de Delpit à mon Premier Ministre encore une fois. Ce garçon-là est réellement malheureux. Taillon m'a informé qu'il avait écrit pour une autre combinaison: ce serait de vous donner Dionne, de la bibliothèque, - si toutefois celui-ci veut quitter son poste, -

9 Ibid., 20 mai 1895. 
et il mettrait Faucher à la bibliothèque ici, afin d'économiser par là le traitement de $\$ 1,400$. que Faucher reçoit pour remplir une charge qui n'a besoin d'aucun titulaire. De sorte que nos plans sont démolis.

Ce pauvre garçon commence à se décourager ayant femme et enfant à supporter. Je l'ai fait venir à Québec, M. Taillon et M. Nantel m'ayant donné à entendre qu'ils pourraient lui donner de l'emploi jusqu'à ce qu'une vacance survienne ${ }^{10}$.

Ce bref intermède terminé, l'analyse de la situation politique à Ottawa se poursuivait sous la plume de l'infatigable DeCelles :

Au moment où je t'écris le sort du gouvernement se discute et se décide: il ne devrait pas être douteux, la majorité ayant accepté sa déclaration l'autre jour. Mais les députés, pris entre leurs liens de parti et la crainte de leurs électeurs, sont indécis, vacillants. La confiance fait défaut en la déclaration ministérielle, et lorsque ce ciment des partis constitués comme les nôtres, a été émietté, effrité par des manques de paroles antérieurs, il vaut mieux briser. C'est une révolution suprême qui, comme tous les oui \& les non irrévocables, coûte à prendre. La situation est pénible pour tout le monde et l'on ne rencontre que des visages anxieux. Seul Angers, que je viens de voir, m'a l'air plus alerte, plus in trim que je ne l'ai vu depuis son arrivée ici. Caron, qui s'était fait un masque souriant et faux, pour traverser la première partie de la crise, car il est diablement mal à l'aise, a l'air soucieux d'un cavalier dont le cheval va buter. Ouimet a regretté, dit-on, d'avoir retiré sa résignation [démission]. Il a filé samedi à Cacouna, ce qui embête Caron ... I M'est avis qu'ils auraient dû résigner [démissionner] et mettre le gouvernement à terre; je ne voyais qu'un danger à cela: la formation, sans Haggart et Montague, d'un parti protestant qui aurait pu former une majorité de gouvernement. Dirigeant les élections que lord Aberdeen n'aurait pu leur refuser, ils en seraient peut-

10 Chapleau à DeCelles, 27 mai 1895. 
être sortis vainqueurs. On ne sait jamais ce que nous réserve le peuple. Il est vrai que Haggart et Montague auraient eu mauvaise grâce de crier contre les catholiques après avoir adopté le principe du redressement de leurs griefs, mais ils ont tant d'audace et, durant les agitations, les paroles dites les semaines avant comptent pour si peu.

Nous causions jadis, à Ottawa, toi et moi, des perspectives du parti, après l'affaire des Jésuites, et nous disions que sir John était le seul lien de cohésion entre les deux sections et que lui disparaissant, elles s'éloigneraient l'une de l'autre par la pente naturelle de leurs idées. Je croyais à la dislocation pour un temps moins éloigné que celui d'aujourd'hui. Mais l'heure de la rupture est arrivée, irrévocablement. Les tories et les bleus se regardent depuis longtemps un peu comme des chiens de faience et, comme dit le duc de Broglie, au sujet de l'alliance de Louis XV et de Frédéric, qui se faisaient des reproches mutuels: "Quand on en est là, entre compagnons d'armes, quand des griefs qui se répètent chaque jour sont des deux parts également motivés, c'est un indice certain que ni intérêts ni sentiments ne s'accordent plus, l'alliance a fait son temps et que les anneaux usés sont prêts à se rompre." Tu vas rire, tu vas m'accuser de vouloir faire de la haute politique. Non, j'ai lu ça, l'autre jour, et il m'a semblé que ces lignes s'appliquaient à la situation.

J'aimerais bien mieux prendre la clef des champs que de faire de la politique en chambre. Les dômes de verdure de Spencer Wood, tes pelouses ouatées d'herbes tendres parlent bien plus à mon imagination que la coupole de la bibliothèque, je t'assure. Tu as l'amabilité de m'inviter à te voir dans ton nid. Merci, je n'y manquerai pas, si la rage de paroles dont sont pris nos députés peut se pasteuriser avant de moudre [sic ?] ${ }^{11}$.

Le 18 août 1895, Chapleau invitait DeCelles à faire un voyage de cinq semaines avec lui en Colombie-Britannique ${ }^{12}$.

11 DeCelles à Chapleau, [ ? ] juillet 1895.

12 Chapleau à DeCelles, 18 août 1895. 
On ignore le but de ce voyage, - sans doute de pur agrément, et on ignore également si DeCelles accepta cette invitation.

Quelques mois plus tard, DeCelles poursuivait son analyse implacable de la décomposition irréversible du parti conservateur:

... Nos pauvres ministres sont en train de transformer leur barque en pétrin; ce qui n'est pas le moyen de s'attirer des compagnons de voyage. Je n'ai rien vu de ma vie de si mal conduite que leur affaire, ou si peu conduite. Il n'y a jamais un coup de barre de donné pour imprimer une direction, mais que du tâtonnement. Ouimet me disait l'autre jour qu'une affaire décidée était revenue quatre fois devant le conseil chercher une nouvelle décision. Et ce n'est pas une nouvelle isolée; c'est la règle générale. Ce n'est pas la politique de Je m'en sacre de Ouimet qui refera la situation. Il est parti pour New-York au moment où il serait nécessaire d'agir vivement à Jacques-Cartier, en étouffant Cousineau. Tout va de mal en pis, faute d'un esprit dirigeant, et ce n'est pas la tête du père Bowell qui loge ce saint Esprit. Cela ne l'empêche pas de se croire plus fin que ne l'était feu sir John, comme il l'a laissé entendre à Tupper l'autre jour en revenant de Washington. Il lui disait que la réputation de finesse de sir John était surfaite: qu'il avait fait bien des choses que lui aurait évitées! (Cela soit dit entre nous). Est-ce croyable que les grandeurs puissent tourner la tête à un homme à ce point ?

Tout de même, je ne puis voir sans un immense chagrin la débâcle qui se prépare et la ruine de ce qui a été le parti conservateur qui a fait de grandes choses. Il est encore vivant, les éléments de force sont là, mais la défaite va les éparpiller, car le lien de cohésion, le ciment manque pour tenir ces forces ensemble. Il sortira de la catastrophe un autre parti conservateur, mais ce ne sera pas celui que nous aurons connu. Ce sera un parti rétrograde, avec Landry, Belleau pour inspirateurs, et le roman de Tardivel pour évangile. Je remarque une tendance rétrograde dans le camp, si rétrograde que nos amis tes anciens fidèles se rangeraient du côté 
de Laurier plutôt que de se mettre dans cette cohorte dont on offrira le commendement à Angers. L'acceptera-t-il, avec son drapeau ? Advenant une débâcle, Ouimet disparaîtra et Caron sera forcé, j'en ai peur, de le suivre, et dire qu'il suffirait de quelqu'un, d'une main ferme pour tout sauver! Je trouve la situation du gouvernement encore meilleure que celle de l'opposition, pourvu qu'on ne continue pas à laisser la débande [débandade ?] se faire. Je suis peut-être pessimiste, mais je ne vois guère de gens qui ne s'alarment pas à la vue de ce qui se passe... ${ }^{13}$

Il y avait certes de quoi s'alarmer, si l'on en juge par cette autre lettre du début de 1896, dans laquelle on a l'impression d'assister aux derniers sursauts d'agonie d'un gouvernement moribond :

... quoique cela puisse t'étonner, il y a encore dans le monde des gens prêts à faire partie d'un cabinet, même moribond... Il y a même des gens qui regrettent de s'être laissé couper le cou de bonne grâce. Ils avaient chacun une gueule de bois vendredi dernier. C'est tout de même curieux ces coins de la comédie humaine. Caron, qui est ficelle en diable \& à qui manque le flair qu'il croit avoir, ne prévoyait pas le dénouement qui l'a mis dans le rang. Jeudi matin, il me disait: "Croyez-vous que je puisse servir sous Angers ?"' Il attendait, c'est évident, un encouragement à ce sacrifice. Je me disais, à part moi, si Angers, comme on le dit, a carte blanche pour reconstituer son coin du cabinet, il ne peut pas s'acoquiner à sir A. P. [Caron] qu'il ne peut sentir et qu'il a démoli à Québec... Il y a des dessous bien curieux à étudier dans ce dernier chambardement. Figure-toi que sir Charles a déclaré à Caron, qui me l'a conté, que ce sont les bolters du mois de janvier qui ont exigé le retour d'Angers. Ça ne me paraît guère croyable après les événements de l'été dernier, mais sir Charles l'a affirmé... Si c'était vrai, les bolters ignorent le tour qu'Angers leur a joué en indiquant à lord Aberdeen le moyen de dénouer la crise du début de la session. Caron parle de se remettre à son bureau

${ }^{13}$ DeCelles à Chapleau, 15 nov. 1895. 
et surtout de s'occuper beaucoup de la Minerve pour en faire le plus important, le plus lu des journaux canadiens. Il préfère se dévouer à cette œuvre que de devenir le successeur de Tupper à Londres. On lui a offert cette succession. Laissons-le réfléchir quelques jours et il aura bientôt fait de chanter son air de bravoure... Le coup de balai l'attend au moment où il s'est donné un mal atroce pour réussir à régler l'affaire des écoles [du Manitoba]. Depuis six mois, lui \& Ouimet ont fait une belle lutte qui devait leur valoir autre chose que la canne [?] du départ \& la feuille de route inattendue ${ }^{14}$.

Dans cette même lettre, DeCelles parle pour la première fois, de son premier livre publié, Histoire des États-Unis. "Quelle affaire que celle de publier un livre ${ }^{15}$ !" Surtout à cette époque, ce devait être toute une entreprise. Quelques mois plus tard, paraissait, dans la Gazette de Montréal une critique de ce livre, reprochant à son auteur certaines interprétations trop favorables aux Canadiens français, au détriment des Américains. D'autre part, l'auteur anonyme de cette critique louait DeCelles de la clarté de son style, en ces termes: "His style is excellent, and he has kept perfectly before his view the old French proverb", "Ce qui n'est pas clair n'est pas français". Là-dessus, il donnait le conseil suivant à ses propres concitoyens, conseil qui pourrait encore être suivi avec profit de nos jours: "It would be well if many writers in English would condescend to remember the teaching of the saying and not become obscure in their effort to reduce fine writing ${ }^{16}$."

Malgré son premier succès littéraire, qui avait eu des échos jusqu'en France ${ }^{17}$, DeCelles n'en continuait pas moins à tenir fidèlement son ami au courant de la déconfiture imminente du gouvernement conservateur à Ottawa. "Ouimet s'est empêtré au dernier caucus [du conseil des ministres] d'une façon lamentable... Le lendemain, il était dégoûté de la politique et parlait

14 Ibid., [ ? ] 1896.

15 Ibid.

16 The Montreal Gazette, 14 juillet 1896.

17 Le Courrier de la Presse, Paris, 1er octobre 1897. 
de s'en aller ${ }^{18}$." Certains conservateurs commençaient même à vouloir se rapprocher de Laurier, dont l'étoile ne cessait de monter: "Landry fait des mamours à Laurier et une de ses amies me disait qu'il avait promis son concours pour les élections au chef de l'opposition ${ }^{19}$." Il n'est pas étonnant que DeCelles pût conclure: "On pense que la majorité du gouvernement ne sera que de quatre ou cinq voix, - si majorité il y a ${ }^{20}$."

Dans cette conjoncture, DeCelles voyait encore le salut du parti conservateur dans Chapleau. Cette confiance d'ami est touchante et même si elle pût être fondée, elle nous paraît, avec le recul du temps, légèrement naĩve. Lisons plutôt DeCelles:

... il n'y a rien de bien neuf ici à part les intrigues qui se renouvellent sans cesse. Les libéraux, - et surtout les libérales, car les femmes s'en mêlent diablement, - ont organisé un système de petite conspiration pour monter le vieux bonze Bowell contre ses collègues ... Personne ici ne paraît savoir où tout cela mènera et où l'on va ... . Angers, que je vois quelquefois, n'a pas confiance: son copain Landry a été vu avec Laurier; c'est Caron qui me l'a assuré, et Beausoleil se promène avec Ouimet. Tout se passe comme si chacun se cherchait des alliances. Angers me disait l'autre jour que s'il était libre, il en ferait une avec les libéraux d'Ontario, mais il ne m'a dit en quoi il n'était pas libre de ses mouvements... Angers est au mieux avec les Aberdeen. Je sais de la source la plus certaine qu'il est leur aviseur [conseiller] confidentiel ... Ceci est confidentiel ... Je suis presque seul à le savoir ... il me semble qu'il découle de la situation embrouillée où nous sommes plusieurs nécessités inéluctables. C'est une situation où les questions de parti, de passé politique comptent pour peu de choses. Il s'agit avant tout de trouver un moyen d'opposer un front solide à l'ennemi pour reconquérir le terrain perdu. Cela ne se peut qu'en groupant ensemble les hommes, les plus forts, appuyés par une députation considérable. Si Ouimet revient, il lui faut quarante-cinq

18 DeCelles à Chapleau, 6 février 1896.

19 Ibid.

20 Ibid. 
hommes à la file. Si cela est impossible, qu'il s'efface ou bien s'entende avec Laurier, - ce qui n'est pas praticable. Dans ce cas, Laurier est l'homme indiqué, bien que je n'ai [e] que la plus mince confiance dans son generalship, car il s'est trop affaibli dans la province de Québec dernièrement, avec sa conduite incertaine. Il reviendra avec une majorité, cela est sûr, mais faible à moins qu'il ne fasse luimême les élections avec le pouvoir en moins. Tout bien considéré, et te cherchant un rôle dans tout ceci, je ne vois que la branche d'olivier de St-Lin reverdie, à condition que tu sois le chef de la Province de Québec. C'est ce qui est la pensée de derrière la tête de bien des gens qui n'osent pas l'exprimer.

Il y aurait ta candidature indépendante, qui entraînerait un bon nombre de députés à la suite, mais il faudrait pour cela un fonds d'élection difficile à trouver entre les deux autres. Tu m'as dit que tu voulais attendre, à tout événement, la fin de tes délices de Spencer Wood. Cela te mènerait à 1897 , - c'est bien loin, et tu auras à cette date plus de dégoût pour la politique dont tu dis beaucoup de mal, mais dont tu as été un enfant gâté, - à côté et au regard de tant d'autres. Je suis pour l'action immédiate ${ }^{21}$.

On voit, par cette lettre, que DeCelles est bien de son époque: en effet, ce n'est pas tant le sort du Canada français qui le préoccupe, que celui du parti conservateur. A cet égard, il est extrêmement intéressant de noter que, cette même année-là exactement, soit 1896, un obscur essayiste du nom d'Edmond de Nevers, écrivait les lignes perspicaces que voici sur la vie politique des Canadiens français: "Depuis 1867, notre armée de politiciens provinciaux ressemble par bien des points à un corps de troupes devenu inutile...22." Quelques lignes plus loin, il ajoutait: "La lutte des partis est, pour un bon nombre, un simple sport, pour les autres c'est une spéculation. Et ce sport ridicule

21 Ibid., 27 février 1896.

${ }^{22} L^{\prime}$ Avenir du peuple canadien-français, Collection du Nénuphar, (Fides, Montréal et Paris, 1964), 92. 
accapare toutes les forces vives de la nation [canadienne-française], et cette spéculation ruine notre province ${ }^{23}$."

Ce sport, ce "politiquage", selon le mot de Nevers, aveuglait en effet toute la population canadienne-française, à l'époque. Ceux qui n'en étaient pas aveuglés, comme Chapleau, - peut-on présumer, - en étaient tout simplement dégoûtés. Comment ne pas l'être, en effet, en lisant de telles choses, peu de temps après la victoire des libéraux:

J'ai vu Tarte... Je l'ai trouvé joliment monté sur ses grands chevaux, au sujet de la lutte que Cornellier se propose de lui faire. Il paraît que cet affreux voyou veut le mettre à la question au sujet de ses faits et gestes lorsqu'il était conservateur. Tarte ne pourrait faire de confession sans frapper son Mea culpa sur la poitrine de Caron, de Dansereau et la tienne par ricochet. Il me semble qu'il exagère la chose à dessein, mais enfin je te répète ce qu'il m'a dit. J'en ai touché un mot à Dansereau, qui pourra facilement brider Cornellier. Je ne vois pas là-dedans de quoi fouetter un chat, mais il vaut autant éviter que ton nom soit mentionné... J'ai toujours envie de rire lorsque je vois Tarte dans l'ancien bureau de Langevin. C'est une des plus grandes ironies des choses de ce monde que j'ai vue.

Le ministère Laurier a bien de la façade mais on ne sait pas encore ce qu'il vaut... Tout de même les libéraux sont là pour longtemps. Quand on pense qu'il se trouve des conservateurs qui s'imaginent les déplacer avant deux ans! C'est beau les illusions ${ }^{24}$ !

Le 10 décembre 1896, DeCelles recevait de Louis J.-A. Papineau, fils du grand tribun, une lettre datée du Manoir de Montebello, à propos de son Histoire des Etats-Unis. Tout en professant une vive admiration à l'égard de son livre, Papineau formulait des observations critiques très pertinentes, dénotant une profonde connaissance du sujet ${ }^{25}$.

23 Ibid., 94.

24 DeCelles à Chapleau, 29 septembre 1896.

25 Louis J.-A. Papineau à DeCelles, 10 décembre 1896. 
Le 29 janvier 1897, Chapleau adressait à DeCelles une lettre dans laquelle il lui faisait part d'une fête en l'honneur de sir James LeMoine, lettre qui dénote une délicate attention de la part de Chapleau:

Nous fêtons demain, à Spencer Wood, le nouveau “chevalier" sir James Le Moine. Ne pourriez-vous pas, entre vous, les [gens] d'Ottawa, vous arranger pour lui envoyer une dépêche qui lui serait communiquée pendant le dîner? Tu sais quil est chatouilleux à cet endroit, et c'est un bien brave homme, après tout ${ }^{26}$.

Peu de temps après, DeCelles faisait part à Chapleau de son avis sur le règlement de l'affaire des écoles du Manitoba, en ces termes:

... Depuis que je t'ai vu, la situation politicoreligieuse ne s'est guère améliorée, au contraire, et l'interview de Drolet me paraît avoir versé du pétrole sur le feu. C'est grand dommage, car c'est encore Jean-Baptiste qui va écoper dans ce surcroît d'agitation. Il est fâcheux que tu n'aies pas été en position d'aller à Rome, en passant par Paris, pour déguiser tes démarches. Je pense que tu aurais réglé toute cette malheureuse affaire, où je cherche en vain le St-Esprit ou l'esprit sain. Au point où en sont arrivées les choses, il faudrait, il me semble, l'intervention d'un homme d'autorité pour faire entendre raison aux belligérants. La ligne de partage des opinions entre le gouvernement et l'Eglise de Québec n'est pas considérable: l'un et l'autre veulent rendre justice à la minorité manitobaine, là où le dissentiment commence, c'est lorsqu'il s'agit de la forme de cette mesure de justice. A gauche comme à droite, on perd de vue le but à atteindre, comme cela se produit souvent dans les querelles de famille, faites pour s'entendre et non pour se dire des choses désagréables. Nul [ne] gagnera la cause si Laurier est condamné par un mandement collectif, après l'avoir été par les mandements particuliers des évêques. C'est cela qui arrangera bien les affaires! Quand même le prestige de Laurier serait

26 Chapleau à DeCelles, 29 janvier 1897. 
diminué, et même beaucoup, il en aura toujours assez pour finir son bail de cinq ans. Il n'y a aucun signe que passé ce terme les tories s'attèleront à la grosse entreprise de rétablir les écoles séparées làbas, bien qu'ils soient prêts à avaler les pires couleuvres pour retrouver l'assiette au beurre.

Il me semble que Laurier en se renfermant dans sa dignité a manqué de stratégie. Il n'y a rien comme de parler pour s'entendre, même entre adversaires. Il aurait bien pu sortir de sa tour d'ivoire pour causer avec les évêques. Il avait Mgr Duhamel sous la main, qui aurait pu calmer les autres. Il faut avouer qu'avec tous les atouts qu'il avait en main, avec la faculté de jeter tous ses collègues par-dessus bord, avec la faculté de dire à Greenway: "Crois ou meurs", il a fait peu de choses. C'est Greenway qui a posé les conditions au lieu de les recevoir. Mais l'affaire ayant été bâclée comme cela, il n'y a plus qu'à l'avaler. Et Drolet, il n'a pas poussé vers une solution. Entre nous, il a mis les pieds et ceux de Laurier dans les plats en regrettant de ne pouvoir pas en mettre davantage. Je n'ai jamais vu un pareil gâchis, et je me demande à qui il comptait rendre service, avec toutes ces indiscrétions. Tout cela est bien triste et prépare mal l'avenir ${ }^{27}$.

Quelques mois plus tard, Chapleau offrait à DeCelles un siège au Conseil législatif de Québec, dans les termes suivants:

Ce dont nous parlions il y a quelque temps et dont tu paraissais t'amuser, je veux dire ta nomination au Conseil législatif, est venu hier sur le tapis d'une manière sérieuse. On a demandé mon consentement pour nommer à cette position différentes personnes dont on m'a soumis les noms. Or comme j'avais prévenu le Premier Ministre, avant les élections, que, s'il voulait faire des nominations à des postes importants, il devait me les soumettre avant la votation, pour s'épargner et m'épargner des ennuis, je lui ai dit que je ne croyais pas devoir sanctionner les nominations qu'il me proposait et qui était tout à fait nouvelles pour moi. J'ai eu, à ce sujet, des récriminations assez désagréables, non

27 DeCelles à Chapleau, 6 mars 1897. 
pas de Flynn, dont la correction a été parfaite, mais de quelques autres, que je ne veux pas nommer aujourd'hui, toujours les mêmes.

Or il arrive que ton nom, qui m'avait été soumis, dans le temps, par Nantel, pour la succession de Dorion, m'a été de nouveau proposé. J'ai répondu que le cas était bien différent, que j'avais déjà donné mon approbation à la nomination et que, si l'on me faisait la proposition régulièrement, je donnerais mon assentiment. J'ai ajouté qu'il serait nécessaire de connaître d'abord tes vues à ce sujet; je savais ce que tu m'avais dit, et ne voulais pas que ta nomination fût faite sans ton acceptation. Je savais, du reste, que cette nomination ne serait pas désagréable à Laurier et ne soulèverait de récriminations que dans les petites gazettes radicales.

...je t'écris moi-même, pour savoir si tu as changé d'idée et si tu accepterais aujourd'hui cette nomination... Tout n'est pas rose dans le métier de "cabinet-maker", surtout quand il s'agit de disposer de vieux meubles et les remplacer ...28.

La réponse de DeCelles fut un refus catégorique, dont il s'expliquait dans les termes suivants:

Non, je ne veux pas être conseiller législatif, comme mon télégramme te l'a déjà appris. S'il n'y avait que cette illustre assemblée à contenter et si j'avais du pain sur la planche pour faire de la politique, je n'aurais pas hésité. Mais, sur ma nomination se greffait l'obligation de faire du journalisme pour aider la cause, c'est là le hic. On attendait beaucoup de moi et c'est le meilleur moyen de se ménager des désappointements aux uns et des ennuis aux autres. On n'est jamais sûr de plaire dans ces sortes d'emtreprises. Le père Titi [?] m'a appris ce que c'est que de rédiger un journal pour un autre. Je redouterai [s] une pareille calamité qui serait irréparable, car la perspective politique n'offre pas aujourd'hui de retraite en perspective aux journalistes conservateurs.

Les autres raisons qui m'attachent ici, je les passe sous silence: tu les connais comme moi. L'âge

28 Chapleau à DeCelles, 17 mai 1897. 
qui s'avance, la foi qui s'en va [foi politique plutôt que religieuse, semble-t-il], le scepticisme qui vient, - je devrais dire: arrive.

Les libéraux ici ont l'embarras de trop de pouvoir. Tarte me le disait hier et Pelletier il y a trois jours. Si j'ai bonne mémoire, je t'écrivais l'automne dernier, qu'une victoire des libéraux à Québec serait fâcheuse pour tout le monde. Seulement cela va faire une détente à Ottawa en ce qui concerne les places.

Laurier m'a offert d'aller à Londres et Paris, pour assister au Congrès des bibliothécaires. Il ne reste plus que l'ordre en conseil [décret] à passer: je pourrais bien y aller sans cela, mais je veux que la question soit bien définie avant mon départ. Si j'y vais, j'amène femme et enfant. Ne parle pas de la chose: une indiscrétion de journal ferait tout manquer ${ }^{29}$.

On ignore si DeCelles fit ce voyage, car la lettre suivante qui reste de cette correspondance est datée du 15 janvier 1898, donc huit mois plus tard. Elle renferme un passage révélateur de l'ampleur de vues de Chapleau, qu'il convient de citer:

... On ne sait guère à quoi s'en tenir par ce temps de lettres ouvertes et de secrets violés. Au sujet de celle que l'on a volée, ne vaudrait-il pas mieux la publier telle quelle que de la laisser déchiqueter par phrases isolées, en l'accompagnant d'un article montrant que tu as toujours voulu la concentration des forces canadiennes dans le but d'accomplir les réformes qui ne se feront jamais sans cela ${ }^{30}$ ?

La dernière lettre qui nous soit restée de DeCelles à Chapleau est datée du 12 février 1898, soit deux mois après que Chapleau eut quitté Spencer Wood et quatre mois seulement avant sa mort. Cette lettre révèle encore l'attachement inaltérable et la confiance inébranlable que DeCelles vouait à son ami de toujours :

J'allais encore t'appeler gouverneur, par habitude; si tu ne gouvernes plus, tu peux encore régner

29 DeCelles à Chapleau, [?] mai 1897.

30 Ibid., 15 janvier 1898. 
sur un bon nombre de tes anciens sujets. Tu as le loisir de songer quand et comment. En effet, rien à faire pour le présent...

J'ai pensé aller à Montréal ce matin; le mauvais temps m'a découragé. J'ai remis la partie à vendredi prochain le soir pour passer la journée de samedi à la ville. Je voulais te voir et te demander ce que tu pensais de l'idée de faire dans la Presse une série d'articles de haute politique, au-dessus des partis, puis de descendre graduellement au niveau des intérêts présents. C'est effrayant ce que la génération actuelle d'aspirants à la vie politique paraît manquer des premières notions du métier auquel elle se croit appelée! La politique n'a jamais été aussi terre-à-terre qu'aujourd'hui! Nous aurions pu faire le travail en question en commun ... Le Courrier du Canada te prend à partie au sujet de ton entrevue sur la question Tupper. Il est très modéré. On reconnaît la plume d'un de tes ex-ministres. Ne fait-il pas erreur lorsqu'il dit que tu as signé un rapport d'un sous-comité du Conseil [des ministres] qui s'était occupé de la question des écoles [du Manitoba]. Si ma mémoire ne me trompe pas, tu m'as dit avoir refusé de signer ce rapport. A propos des écoles, j'ai appris que Mgr Langevin, Rochon, inspecteur des écoles, et le Dr Bryce ont eu plusieurs entrevues avec Sifton, et Laurier, je crois, relativement à des questions de détails sur le fonctionnement de la loi. On serait à la veille d'un modus vivendi acceptable. Peut-être en sais-tu plus long que moi sur ce point...

Tu ne parles pas de ta santé. J'en conclus que cela va assez bien. Il n'y a pas de raison pour que tu ne reprennes pas le dessus, surtout si tu t'en tiens à ta résolution d'abstinence absolue ${ }^{31}$.

Ainsi se clôt cette correspondance entre DeCelles et Chapleau. Ce dernier, contrairement à ce que croyait son ami, n'était pas bien du tout à ce moment-là. En fait, dès février 1898, Chapleau était atteint d'une crise de néphrite qui devait l'em-

31 Ibid., 12 février 1898. 
porter au mois de juin suivant ${ }^{32}$. D'ailleurs, dès 1881, "il se sentait déjà atteint par la maladie ${ }^{33}$ ". C'est que Chapleau avait abusé, semble-t-il, de sa santé. "Le fait est qu'il ne se ménageait guère. Débordant d'énergie, gai compagnon, adoré des femmes, dîneur artiste, connaisseur des bons vins, populaire dans le monde où l'on fait bonne chère, aimant le jeu, capable de passer des nuits entières autour d'une table verte, les cheveux blanchissant sous la migraine, alternant le travail avec le plaisir, il se fatiguait, usait ses forces et veillissait ${ }^{34}$."

$\mathrm{Au}$ mois de mai 1898, Chapleau revenait d'un voyage de santé à Atlantic City "décharné, rapetissé et plus vieux de vingt ans ${ }^{35}$ ". Vers cette époque, il recevait une lettre de Hugh J. Macdonald l'invitant à revenir dans la politique. Après avoir consulté ses amis, dont l'abbé Colin, il décidait de se reposer deux ans, avant de "rentrer dans la fournaise ${ }^{36}$ ". Mais ses jours étaient désormais comptés. Au début de juin, il dictait à son nouveau secrétaire particulier, Arthur Beauchesne, une longue lettre à Laurier et une autre, le lendemain, à Hector Fabre. Ce furent ses dernières lettres ${ }^{37}$. Vers le 10 juin, Chapleau fit son testament, ses médecins lui ayant dit qu'il était fini. Dans l'avantmidi du 13 juin, il dit à ses intimes réunis autour de lui: "la mort se fait attendre ${ }^{38}$ ". Le même jour il recevait de Rome un câblogramme du pape: "Saint-Père accorde de tout cœur bénédiction sir Adolphe Chapleau "39." Comme sa femme ne pouvait déchiffrer la signature de ce message, à cause de ses larmes, il dit: "Oui, oui, Rampolla, je le connais ${ }^{40}$." A midi, Chapleau entrait en agonie, alors que les abbés Colin et Racicot se mettaient à réciter les prières pour les agonisants. Après être

32 Arthur Beauchesne, Adolphe Chapleau, texte original dactylographié d'un article paru dans la Revue Moderne, livraison du 15 mars 1921, 12-15. (Ce texte m'a été prêté par M. Beauchesne lui-même).

33 Ibid., 5.

34 Ibid.

35 Ibid., $12-13$.

36 Ibid.

37 Ibid.

38 Ibid., 13-14-15.

39 Ibid.

40 Ibid. 
"mort comme un saint 41", selon l'expression consacrée, le premier télégramme de condoléances reçu par ses proches fut celui de sir Wilfrid Laurier.

Quel jugement peut-on porter sur Chapleau, après étude de cette correspondance ? Certes, n'est-il pas question de porter un jugement définitif sur cet homme qui, à maints égards, reste pour nous quelque peu énigmatique, sans doute faute de sources plus abondantes. Mais si l'on s'en tient uniquement à cette correspondance, quelques traits paraissent se dégager de la personnalité de Chapleau. D'abord, un incontestable talent. C'était un homme extrêmement doué, à tous égards. Tous les textes concordent là-dessus. Mais, comme dans le cas de nombre de ceux qui sont extrêmement doués, Chapleau nous paraît avoir été un faible de caractère. Il semble, selon nous, avoir manqué de ténacité.

Fut-il un "véritable homme d'État 42", comme l'affirme Beauchesne ? La question est discutable. Certes, la déception profonde de Chapleau à Ottawa expliquerait bien des choses. Et cette phrase de Chapleau, prononcée dès 1888, que rapporte le chanoine Groulx, expliquerait peut-être tout: "L'influence canadienne-française est nulle dans le cabinet, nulle dans la direction de la politique ${ }^{43}$." Il est d'ailleurs intéressant de constater que cette phrase désabusée de Chapleau coïncide exactement avec ce jugement pénétrant d'Edmond de Nevers, qui date précisément de cette époque: "Il résulte de ce qui précède que, depuis 1867, au lieu de concentrer nos forces, de tirer parti de toutes nos ressources intellectuelles et matérielles, et de jeter les bases d'un développement national propre à nous assurer un avenir brillant sur ce continent, nous avons continué la vie de luttes du passé, tout comme si rien n'avait été modifié dans notre existence... Ce n'est pas seulement une interruption dans notre

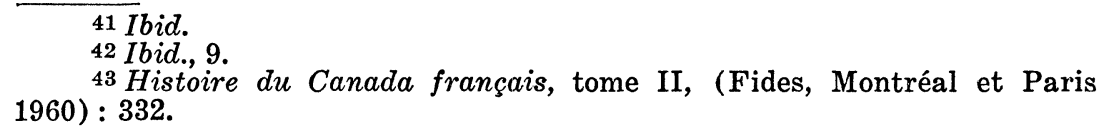


marche que représentent les vingt-cinq dernières années: notre population s'est démembrée, et nous avons fait des pertes irréparables ${ }^{44}$."

Tout compte fait, on peut dire que Chapleau fut victime à la fois de son temps et de l'illusion tenace de pouvoir jouer un rôle valable au sein d'une majorité écrasante. Est-ce là faiblesse de caractère ? Il appartiendra aux générations plus éloignées de la nôtre de démêler cette énigme.

JACQUES GOUIN,

Diplômé en Sciences Politiques

(Ottawa),

Chef adjoint du Bureau des traductions,

Ministère de la Défense nationale

(Ottawa).

${ }^{44} L$ 'Avenir du peuple canadien-français, ibid., 107-108. 